\title{
Ectodermal Dysplasia - A Case Study of Two Identical Sibilings
}

\author{
D. Ayesha Thabusum ${ }^{1, *}$, N. Rajesh ${ }^{2}$, R. Sudhakara reddy ${ }^{2}$, T. Ramesh ${ }^{2}$ \\ ${ }^{1}$ Department of oral medicine and radiology, Drs.Sudha \& Nageswara rao Siddhartha institute of Dental sciences, Gannavaram \\ ${ }^{2}$ Department of oral medicine and radiology, Vishnu Dental College, Bhimavaram \\ *Corresponding author: drayeshaomr@gmail.com
}

Received November 24, 2014; Revised December 04, 2014; Accepted December 07, 2014

\begin{abstract}
The Ectodermal Dysplasia comprises a large, heterogeneous group of inherited disorders that are defined by primary defects in the development of two or more tissues derived from embryonic ectoderm. The tissues primarily involved are the skin, hair, nails, eccrine glands and teeth. The X-linked recessive ED (Christ-SiemensTouraine syndrome) is the most common disorder; it affects males and is inherited through female carriers. It is characterized by the triad of signs comprising sparse hair (atrichosis or hypotrichosis), abnormal or missing teeth (anodontia or hypodontia) and inability to sweat due to lack of sweat glands (anhidrosis or hypohidrosis). The lack of teeth and the special appearance were reported to be major concerns. Two case reports illustrating the typical features of anhidrotic ectodermal dysplasia have been presented. Since there is no definitive treatment for the disease, an early prompt diagnosis and a multidisciplinary approach in planning a treatment can improve the quality of life of the patient.
\end{abstract}

\section{Keywords: Christ-Siemens-Touraine syndrome, hypotrichosis, hypodontia, hypohidrosis}

Cite This Article: D. Ayesha Thabusum, N. Rajesh, R. Sudhakara reddy, and T. Ramesh, "Ectodermal Dysplasia - A Case Study of Two Identical Sibilings.” International Journal of Dental Sciences and Research, vol. 2, no. 6 (2014): 175-178. doi: 10.12691/ijdsr-2-6-14.

\section{Introduction}

Ectodermal dysplasias, as first described by Thurnam in 1848 are a heterogeneous group of disorders characterized by developmental dystrophies of ectodermal structures [1]. About 192 clinically and genetically distinct ectodermal dysplasia have been catalogued [2]. This X-linked recessive disorder affects males and is inherited through female carriers.

The incidence of this condition is $1: 100,000$ births in males and the carriers incidence is probably around 17.3 in 100,000 women [3]. Ectodermal Dysplasia is characterized by the triad of signs comprising sparse hair (atrichosis or hypotrichosis), abnormal or missing teeth (anodontia or hypodontia) and inability to sweat due to lack of sweat glands (anhidrosis or hypohydrosis) [4].

Depending on the number and functionality of the sweat glands, two major types of ectodermal dysplasia's have been classified which include; (1) X-linked anhidrotic or hypohydrotic type - Christ-Siemens-Touraine syndrome. (2) Hidrotic type clouston's syndrome. ChristSiemens-Touraine syndrome is the most frequently reported manifestation of ectodermal dysplasia [5].

Freire-Maia and Pinheiro proposed the first classification system of the ectodermal dysplasias in 1982, with additional updates in 1994 and 2001 [6]. Overall the ED were classified into either group A disorders, which were manifested by defects in at least 2 of the 4 classic ectodermal structures and group B disorders which were manifested by a defect in one classic ectodermal structure in combination with a defect in one other ectodermal structures (i.e.ears, lips).The most common classification of ectodermal dysplasia include Hypohidrotic (anhidrotic) (HAED) and Hidrotic ED(HED) [2].

When at least 2 types of abnormal ectodermal features occur, such as malformed teeth and extremely sparse hair, the patient is diagnosed with ectodermal dysplasia syndrome [7]. The diagnostic tool is typical clinical physionomy. The lack of teeth or abnormal teeth and the special appearance were reported to be of major concerns.

Oral traits of ectodermal dysplasia may be expressed as anodontia or hypodontia, with or without a cleft lip and palate. Other special features or appearances include frontal bossing, thick and protuberant lips, broad depressed nasal bridge of nose, peri-oral and peri-orbital pigmentation and deformed ears [2-8].

Here with, we report series of cases with typical Xlinked recessive hypohidrotic Ectodermal Dysplasia of two identical sibilings.

\section{Case Study}

A 13 year and 14 year old female patients reported to the Department of Oral Medicine, Oral Diagnosis and Radiology with a chief complaint of missing teeth in upper and lower arches since childhood. Patient reports of no history of exfoliation of teeth in the maxillary and 
mandibular arches except in the upper anterior region where the deciduous teeth were replaced by the existing permanent teeth (Figure 1).

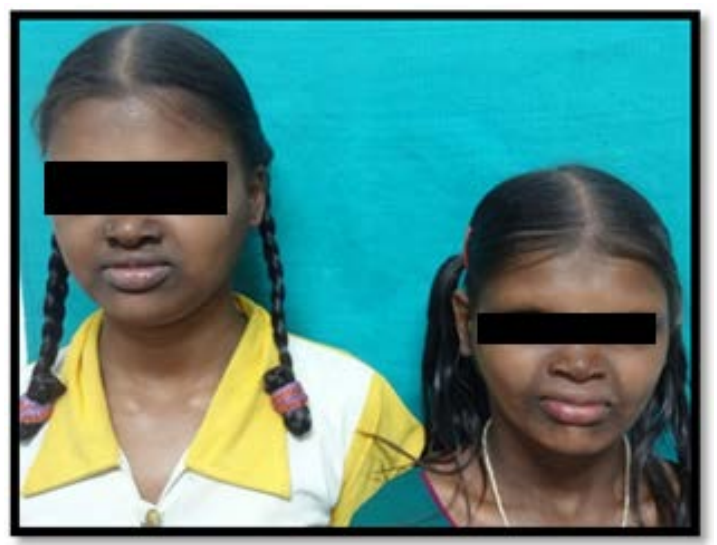

Figure 1. Two siblings aged fourteen and thirteen

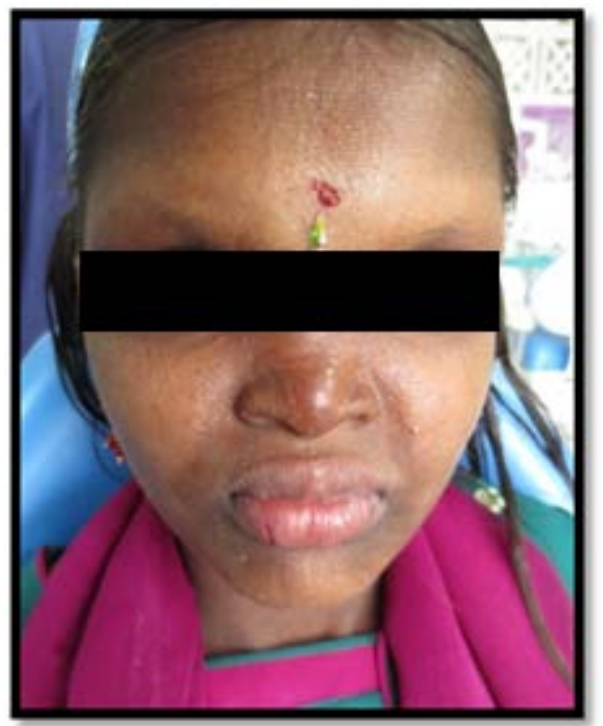

Figure 2. Two siblings presented with the same features of Ectodermal dysplasia

Intra oral examination revealed complete absence of teeth in the mandibular arch and presence of conical shaped right and left maxillary central incisors $(11,21)$. Further intra oral examination revealed loss of sulcus
Patient reports no history of trauma either in the past. The lack of primary and permanent teeth in the oral cavity resulted in dietary and speech problems. Patient also complains of dry skin and dry mouth with difficulty in swallowing.

The parents further reported a positive family history wherein history of consanguineous marriage and also no evidence of similar afore mentioned features either in the maternal or paternal side of the patients is observed. Both the children had normal intelligence and no visual and auditory problems.

During extra oral examination, the patients exhibited the typical features of ectodermal dysplasia: frontal bossing, thick and protuberant lips, saddle nose, soft, dry and light colored skin on the face and on the upper and lower extremities, increased pigmentation around the ala of the nose, sparse hair on the scalp, eye brows and eye lashes; dry lips ; peri oral and peri orbital pigmentation (Figure 2).

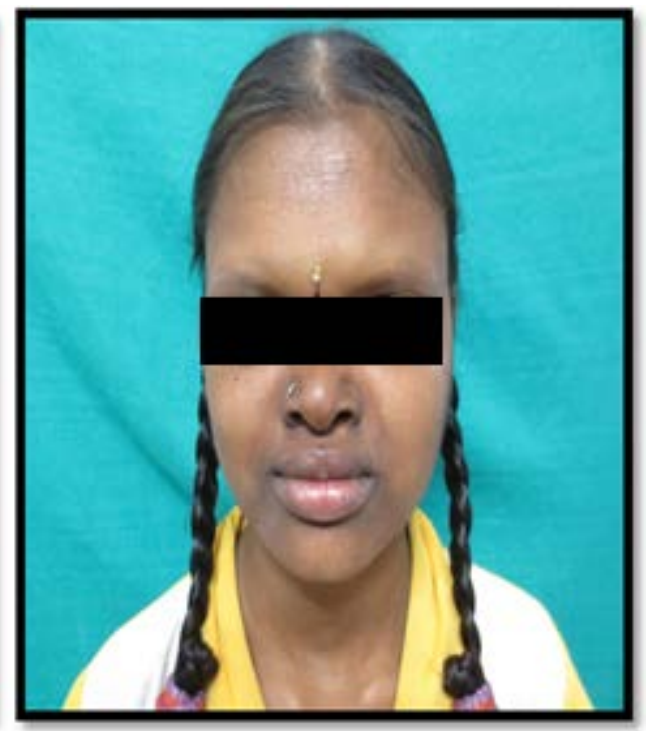

depth in the entire mandibular arch and maxillary arches except in maxillary anterior region. Thinning of alveolar ridges evident in both maxillary and mandibular arches. (Figure 3).


Figure 3. Two siblings presented with conical shaped incisors and absence of remaining teeth

Patient is subjected to radiographic examination. Orthopantomograph examination confirmed the absence of teeth in mandibular arches and presence of two teeth $(11,21)$ with under developed alveolar ridges (Figure 4). 



Figure 4. Orthopantomographs of two siblings

\section{Discussion}

Ectodermal dysplasia is a congenital, diffuse and non progressive disorder. Though Thurnam was the first person to describe the condition, the term ectodermal dysplasia was coined by Weech in 1929 [2].

Most of the patients with Ectodermal Dysplasia have a normal life expectancy and normal intelligence. The siblings in the present case reported too, have normal intelligence with no visual and auditory disturbances. All the ectodermal dysplasias appear to be genetic in etiology. The recent cloning of the gene has led to the identification of a novel transmembrane protein "ectodysplasin A" and "ectodysplasin A receptor" genes ${ }^{1}$. Genetic studies regarding the etiology of Ectodermal Dysplasia reveal that mutations in these genes are responsible for $\mathrm{X}$-linked and autosomal hypohidrotic Ectodermal dysplasia.
The diagnosis of Ectodermal Dysplasia is made when atleast two types of abnormal ectodermal features such as malformed teeth and extremely sparse hair are present $[5,6,7]$. Missing teeth or delay in teething often starts to worry the parents and leads the clinician to the diagnosis of ectodermal dysplasia. It is a well known fact that dental findings in ED may range from hypodontia / oligodontia to anodontia of the primary or permanent teeth. However, the congenital absence of primary teeth is relatively rare [4].

Nevertheless, complete anodontia involving primary and permanent dentitions was evident in both the maxillary and mandibular arches except in the maxillary anterior region in both the present cases. In the present case scenario the maxillary deciduous right and left central incisors $(11,21)$ are replaced by the existing permanent incisors.

The literature documents that there are generally more teeth in the maxilla than the mandible, although both the 
jaws can be toothless [9]. The present case series clearly depicts the presence of maxillary teeth and complete absence of mandibular teeth. Besides the delay in teething, the teeth erupted appear to be conical or peg shaped in appearance with upper incisors and cuspids affected more commonly [3]. The presence of conical shaped maxillary incisors in the siblings further strengthens the diagnosis of Ectodermal Dysplasia.

Interestingly, in both the cases the growth of the jaws are not affected, however resulted in underdeveloped alveolar ridges as witnessed in radiographic examination (orthopantomograph) This is attributed to the fact that, in the absence of teeth the alveolar process do not develop much resulting in reduction of normal vertical dimension.

Phenotypic tests such as assessment of sweating and dental findings aids to identify possible female carriers. Two methods of assessment of sweating have been developed: The first sweat test is performed on the backs of the carrier female and gives a V-shaped pattern of streaks that refers to the lines of Blaschk. The other method is to make counts of the sweat pores along ridges of the finger tips or palms. Sweat pore counts using yellow starch iodine, pilocarpine iontophoresis may document hypotrichosis [10].

Combination of both dental examination and sweat testing enhances clearly the chances of making a correct diagnosis of identifying female carriers. Dental radiographs can provide useful additional information and can be a simple screening test for carrier states. Alopecia areata focal dermal hypoplasia incontinentia pigmenti, and dyskeratosis congenita are some of the conditions through which the differential diagnosis is made $[11,12]$.

So, based on all the above possible clinical and radiological findings found in both the siblings, a diagnosis of X-linked hypohidrotic ectodermal dysplasia has been arrived.

\section{Conclusion}

The clinical manifestations of ectodermal dysplasia pose considerable social problems in the affected individuals and hamper the quality of life of the patient. So, the role of an oral physician is challenging and plays a pivotal role in arriving at an early and prompt diagnosis of such congenital disturbances. A multidisciplinary approach is necessary in planning a treatment and thus ultimately benefiting the patient. Oral rehabilitation remains the mainstay of treatment part as far as the dental considerations are concerned. The analysis of the function of the genes and the effects of gene mutations will be a promising challenge and opens a new vista in the development of therapeutic methods.

\section{Ethics Statement}

The consent of the patient was sought prior to and approved the inclusion of case and their photographs.

\section{References}

[1] Tarjan I, Gabris K, Rozsa N. Early prosthetic treatment of patients with ectodermal dysplasia: a clinical report. J Prosthet Dent 2005; 93: 419-424.

[2] Jananee J, Satish Kumar M, Balaji Sumathi. Ectodermal Dysplasia - A case report. Indian Journal of Multidisciplinary Dentistry 2012; 2(2):465-467.

[3] Mortier K, Wackens G. Ectodermal Dysplasia anhidrotic. Orphanet Encyclopedia. September 2004.

[4] Bani M, Tezkirecioglu A M, Akal N, Tuzuner T. Ectodermal Dysplasia with Anodontia: A report of two cases. European Journal of Dentistry 2010; 4: 215-222.

[5] Viera K A, Teixeira M S, Guirado C G, Gaviao M B. Prosthodontic treatment of hypohidrotic ectodermal dysplasia with complete anodontia: case report. Quintessence Int 2007; 38: 75-80.

[6] Freire-Maia N, Lisboa-Costa T, Pagnan NA. Ectodermal dysplasias: how many?. Am J Med Genet. 2001; 104(1): 84.

[7] Yavuz I, Ulku S Z, Unlu G, Kama J D, Kaya S, Adiguzel O, Kaya FA, Tumen EC, Zortuk M, Bhasi E, Arslanoglu Z.Ectodermal dysplasia: clinical diagnosis. Int Dent Med Disorders 2008;1:1-10

[8] Geetha Paramkusam, Venkateswarlu Meduri, Lakshmi Kavitha Nadendla, Namratha Shetty. Hereditary Hypohidrotic Ectodermal Dysplasia: Report of a Rare Case. Journal of Clinical and Diagnostic Research. 2013; 7(9): 2074-2075.

[9] Aswegan A.L., Josephson K.D., Mowbray R., Pauli R.M., Spritz R.A., Williams M.S.: Autosomal dominant hypohidrotic ectodermal dysplasia in a large family. Am. J. Med. Genet. 1997; 72: 462-467

[10] Clarke A., Burn J: Sweat testing to identify female carriers of Xlinked hypohidrotic ectodermal dysplasia. J Med Gen 1991(28): 330-333.

[11] Lowry R B, Robinson G C, Miller J R. Hereditary ectodermal dysplasia. Symptoms, inheritance patterns, differential diagnosis, management. Clin Paediatric 1966; 5: 395-402.

[12] Prerna Taneja Mathur, Rahul Paul, Priyank Banthia, Tapasaya Juneja Kapoor Understanding Ectodermal Dysplaisa. Indian Journal of Dental Sciences 2013 Supplementary Issue, Issue:4, Vol.: 5 . 